Reprod. Nutr. Dévelop., 1988, 28 Suppl. $\mathrm{n}^{\circ} 1,61-62$

\title{
Prévision de la quantité d'herbe ingérée par la vache laitière au pâturage. Comparaison de méthodes d'estimation de la digestibilité et du mode d'échantillonnage des fécès
}

\author{
R. BISTON, Nicole BARTIAUX-THILL, A. THÉWIS ( ${ }^{1}$ ), E. FRANÇOIS, P. LIMBOURG, M. \\ GIELEN $\left({ }^{2}\right)$, P. DARDENNE
}

Centre de Recherches agronomiques de l'Etat, avenue de la Faculté d'Agronomie, 22, B - 5800 Gembloux, Belgique.

(') Faculté des Sciences agronomiques de l'Etat, passage des Déportés, 2, B - 5800 Gembloux, Belgique.

( $\left.{ }^{2}\right)$ Centre de Recherches sur I'Elevage et les Productions fourragères en Haute Belgique (IRSIA). rue des Vétérinaires, 56, B - 1070 Bruxelles, Belgique.

Summary. Herbage intake of grazing dairy cows was estimated with several techniques. Grab sampling introduced an upwards bias in the estimate of faecal output comparison with field sampling. Estimates of OM digestibility by NIR and faecal indexes methods were similar except with high level of concentrates.

La présente étude a pour objectif une comparaison de divers processus d'échantillonnage des fèces dans le rectum ou sur le pré et de différentes méthodes de prévision de la digestibilité (azote fécal, lignine fécale, spectrométrie de réflexion dans le proche infrarouge).

Matériel et méthodes. L'essai s'effectue dans les Ardennes belges sur 2 lots de 6 vaches Pie Noire pâturant une prairie permanente en rotation: lot I: 5 vaches $/$ ha ; $4,34 \mathrm{~kg}$ MS de concentré/animal/jour; fumure: $150 \mathrm{~kg}$ d'azote (UN)/ha; lot II : 5 vaches/ha ; $2,33 \mathrm{~kg} \mathrm{MS} \mathrm{de} \mathrm{concentré/animal/jour;} \mathrm{fumure} \mathrm{:}$ $250 \mathrm{UN} / \mathrm{ha}$. Les animaux reçoivent journellement, en 2 repas (aux heures de traite), le concentré contenant $20 \mathrm{~g}$ de $\mathrm{Cr}_{2} \mathrm{O}_{3}$ ainsi que des particules colorées permettant l'individualisation des bouses de chaque animal. Les fèces sont récoltées journellement dans le rectum (rectal 30 jours) entre $6 \mathrm{~h} 00$ et $7 \mathrm{~h} 00$ et entre $15 \mathrm{~h} 30$ et $16 \mathrm{~h} 30$ (heures de traite), ou périodiquement pendant 6 jours régulièrement espacés sur les 30 jours d'essai, soit dans le rectum (rectal 6 jours) soit par échantillonnage de toutes les bouses sur la prairie (global 6 jours). La digestibilité de l'herbe est estimée à partir de l'azote fécal (Bartiaux-Thill et Oger, 1986), de la lignine fécale (Thévis et al,, résultats non publiés) ou de la digestibilité in vivo de l'herbe par spectrométrie dans l'infrarouge proche (NIRS) (Biston et Dardenne, 1985). Cet échantillon d'herbe est prélevé à la cisaille, à $2,5 \mathrm{~cm}$ du sol, à l'entrée du bétail sur chaque parcelle, soit 8 fois sur les 30 jours d'essai. Les dosages d'azote et de lignine sont effectués par NIRS.

Résultats et discussion. Par rapport à l'échantillonnage sur le pré, les prélèvements rectaux sous-estiment la concentration journalière en $\mathrm{Cr}_{2} \mathrm{O}_{3}$ de $7,0 \%$ dans le lot I et de $2,7 \%$ dans le lot II pour 6 jours et de $7,0 \%$ et $2,4 \%$ pour 30 jours.

Reproduction, Nutrition, Développement, 28, Suppl. $n^{` 1 / 88-5}$ 
II en résulte des différences importantes $(0,42$ à $0,97 \mathrm{~kg}$ de MO suivant les processus d'échantillonnage) dans l'estimation de la quantité ingérée (tabl. 1). Toutefois l'analyse des composantes de la variance entre animaux, jours et échantillons, montre que la précision des 2 modes d'échantillonnage est du même ordre de grandeur (variance liée à l'échantillonnage lot I: global 0,219; rectal $0,209$; lot II: global 0,138 et rectal 0,266$)$. La variance entre animaux est du même ordre de grandeur que celle liée à l'échantillonnage; par contre la composante de la variance entre jours est faible (0,005-0,065 suivant les lots). Melix et Peyraud (1987) signalent les mêmes phénomènes pour des animaux en stalles ou au pâturage.

Le rapport $\mathrm{Cr}_{2} \mathrm{O}_{3}$ rectal $6 \mathrm{j} /$ rectal $30 \mathrm{j}$ est égal à 1, ce qui permet de simplifier l'échantillonnage des fèces tout en obtenant la même précision dans l'estimation de la quantité de fèces. Le mode d'échantillonnage des fèces n'induit pas de différence dans l'estimation de la digestibilité avec l'N fécal ou la lignine fécale (tabl. 1).

TABL. 1. - Quantité d'herbe ingérée ( $\mathrm{kg} \mathrm{MOl)} \mathrm{en} \mathrm{fonction} \mathrm{du} \mathrm{mode} \mathrm{d'échantillonnage} \mathrm{des} \mathrm{fécès} \mathrm{et}$ de la technique d'estimation de la digestibilité ( $\mathrm{d} \mathrm{MO}$ en \%).

\begin{tabular}{|c|c|c|c|c|c|c|c|}
\hline & & $\mathrm{Cr}_{2} \mathrm{O}_{3}$ & global $6 \mathrm{j}$ & $\mathrm{Cr}_{2} \mathrm{O}_{3}$ & rectal $6 \mathrm{j}$ & $\mathrm{Cr}_{2} \mathrm{O}_{3}$ & rectal $30 \mathrm{j}$ \\
\hline & & $\mathrm{dMO}$ & $\mathrm{MOI}$ & dMO & MOI & $\mathrm{dMO}$ & $\mathrm{MOI}$ \\
\hline \multirow{3}{*}{ Lot I } & $\mathrm{Nf}^{*}$ & 77.9 & 15,10 & 77,4 & 15,82 & 77,1 & 15,60 \\
\hline & $L f^{* *}$ & - & - & 75,5 & 14,52 & 75,6 & 14,59 \\
\hline & DNIR ${ }^{* *}$ & 78,6 & 15,62 & 78,6 & 16,71 & 78,6 & 16,71 \\
\hline \multirow{3}{*}{ Lot II } & $\mathrm{Nf}$ & 77,3 & 12,75 & 77,7 & 13,35 & 77,6 & 13,55 \\
\hline & Lf & - & - & 77,7 & 13,34 & 77.4 & 13,43 \\
\hline & DNIR & 78,1 & 13,16 & 78,1 & 13,58 & 78,1 & 13,83 \\
\hline
\end{tabular}

${ }^{*} \mathrm{dMO}=27,87+22,98 \mathrm{Nf}_{\mathrm{MO}}-2,248\left(\mathrm{Nf}_{\mathrm{MO}}\right)^{2} ; \mathrm{r}=0,97 ; \mathrm{S}_{\mathrm{yx}}=1,35 ; \mathrm{Nf}: \mathrm{N}$. féc.

$* * \mathrm{dMO}: 1,312 \mathrm{Lf}_{\mathrm{MS}}-86,575 ; \mathrm{r}=0,73 ; \mathrm{S}_{\mathrm{yx}}=3,59 ; \mathrm{Nf}=$ lignine fécale.

*** $\mathrm{dMO}=0,984 \times-0,116 ; r=0,95 ; \mathrm{S}_{\mathrm{yx}}=2,0 ; x=$ valeur NIRS.

Les estimations de la digestibilité par les 3 techniques sont identiques pour le lot II. Par contre, pour le lot I, le NIRS donne une valeur légèrement supérieure et la lignine fécale une valeur plus faible. La relation avec la lignine fécale semble plus difficile à employer à cause de son écart-type résiduel élevé $(0,35)$. Quant aux résultats NIRS, leur précision est dépendante de la représentativité de l'herbe analysée par rapport à I'herbe réellement consommée par l'animal. On peut donc conclure que l'azote fécal permet une prévision plus précise que ces deux méthodes.

Bartiaux-Thill N., Oger R., 1986. Grass Forage Sci., 41, 269-272.

Biston R., Dardenne P., 1985. ECC Seminar on Forage digestibity by NIRS. Brussełs 3-4 décembre. Melix C., Peyraud J. C., 1987. Reprod. Nutr. Dev., 27, 217-218. 